\title{
The Research on Longitudinal Flying Quality of Small Unmanned Aerial Vehicle
}

\author{
Zhian Yan, Hudan Sun \\ China Electronics Technology Group Corporation Special Mission Aircraft System Engineering Co., Ltd., Chengdu, China
}

Email address:

yanza@cetca.net.cn (Zhian Yan), sunhd@cetca.net.cn (Hudan Sun)

To cite this article:

Zhian Yan, Hudan Sun. The Research on Longitudinal Flying Quality of Small Unmanned Aerial Vehicle. Automation, Control and Intelligent Systems. Vol. 5, No. 2, 2017, pp. 29-32. doi: 10.11648/j.acis.20170502.12

Received: March 5, 2017; Accepted: April 17, 2017; Published: April 21, 2017

\begin{abstract}
Due to the lack of specific flying quality criteria for UAV (Unmanned Aerial Vehicle), most researchers use the human flying quality criteria to evaluate the UAV flying qualities. This paper, through analyzing the flying performance of the small UAVs before and after the inclusion of the control augmentation systems, has validated the applicability of human flying quality criterion such as CAP and bandwidth criterions to small UAVs and proposed a means of evaluating the flying quality research of UAVs. The research of this paper can provide a reference for the future research of UAV flying quality and help for the design of UAV as well as its flying control system.
\end{abstract}

Keywords: UAV, Control Augmentation System, Flying Quality, Bandwidth Criterion

\section{Introduction}

In recent years, more and more UAVs are used in civil and military areas because of their special advantages. In contrast to the big development of UAVs, the research of the UAV flying quality is badly lagged behind. Until now, there is no specific flying quality criterion for UAVs. As we all know, the human flying quality criterion is the design reference and standard for human aircrafts and the control system, and the flying quality criterion can ensure the security and reliability of the aircraft, enhance the efficiency of the aircraft design, and reduce the design period and cost. The mature human flying quality research promoted the quick development of the human aircrafts. Unfortunately, there is no specific UAV flying quality criterion domestic and abroad. All the present researches of UAV flying qualities have been undertaken according to the human flying quality criterion. And more seriously, there is no definitive proof that the human flying quality criterion can be used to evaluate the UAV flying quality, and this is the hot research topic now. The references [1-6], [10-12] analyzed the applicability of human flying quality criterion for UAVs, but the conclusions are different and even contradictory.

As there is no special UAV flying quality criterion, this paper analyzes the flying performance of the small UAV before and after the inclusion of control augmentation system and evaluates the longitudinal flying quality using human flying quality criterion. Through the research of this paper, we conclude some new properties of the flying quality for small UAV and propose a means to evaluate the longitudinal flying quality research of small UAV.

\section{Flying Performance Validation with/Without Augment System}

The research UAV example is a small UAV about $5 \mathrm{Kg}$, with maximum flying altitude of $2000 \mathrm{~m}$, maximum flying speed of $0.06 \mathrm{Ma}$. We choose the design point $(\mathrm{h}=1000 \mathrm{~m}$, $\mathrm{Ma}=0.05)$ to evaluate the flying performance of the small UAV. We analyzed the time-domain response performance of the UAV with and without control augment system, as following:

(1) The time-domain response comparison of pitch angle and pitch rate between the UAV with and without control augment system, as "Figure 1" displaying: 

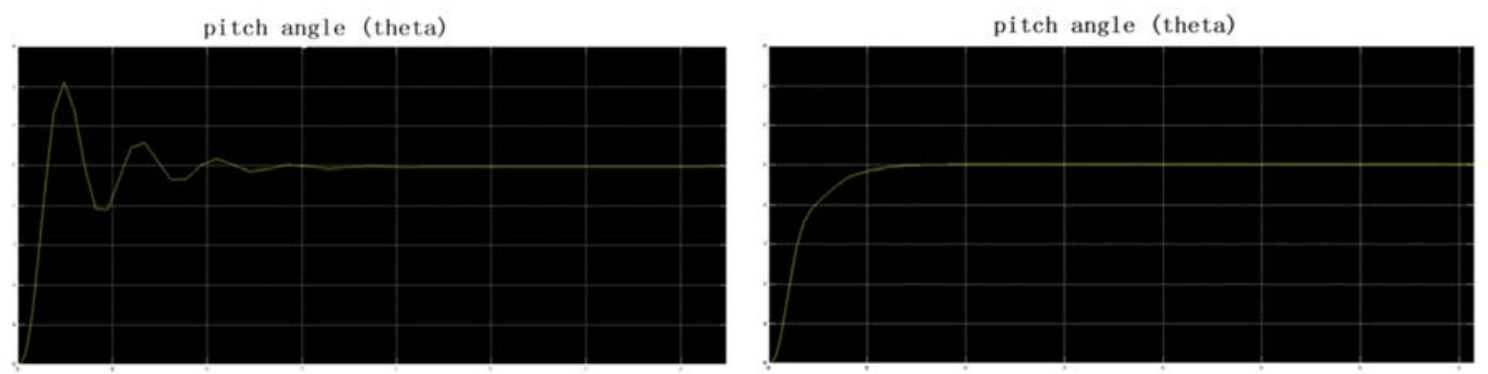

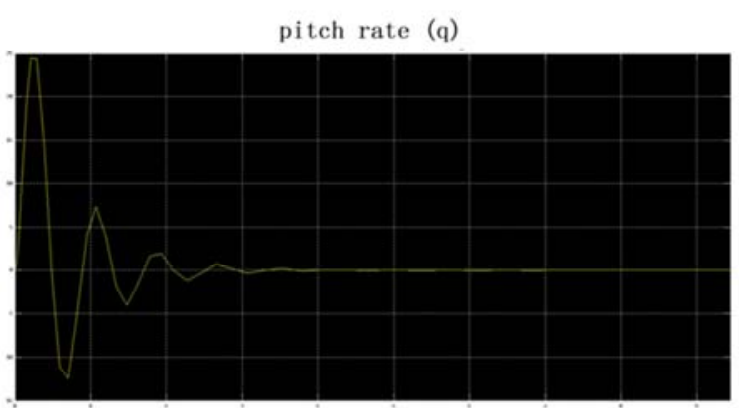

without control augment system

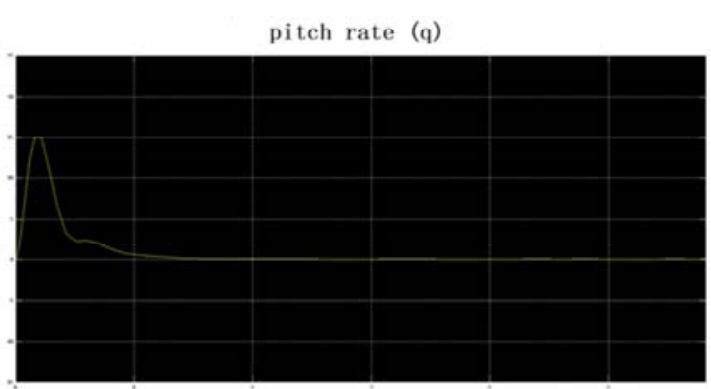

with control augment system

Figure 1. Time-domain response comparison.

(2) The frequency-domain stability comparison between the UAV with and without control augment system, as "Figure 2" displaying:

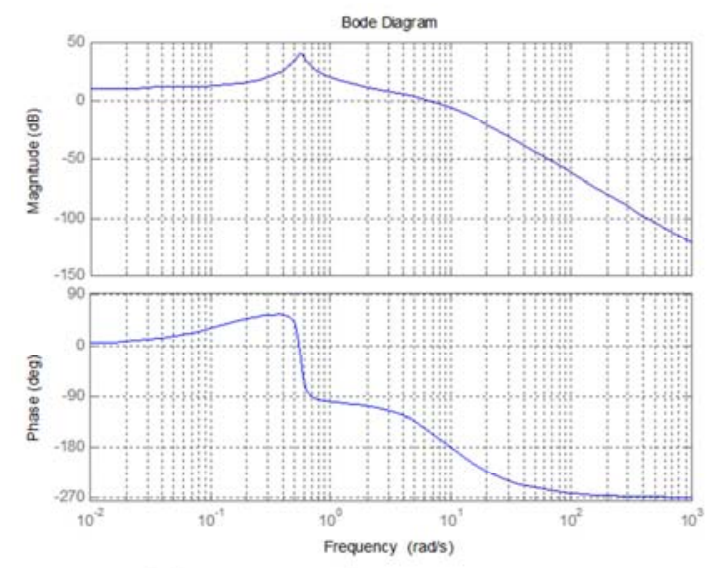

without control augment system:

$$
\begin{aligned}
& \mathrm{Gm}=6.2 \mathrm{~dB} \\
& \mathrm{Pm}=30.4^{\circ}
\end{aligned}
$$

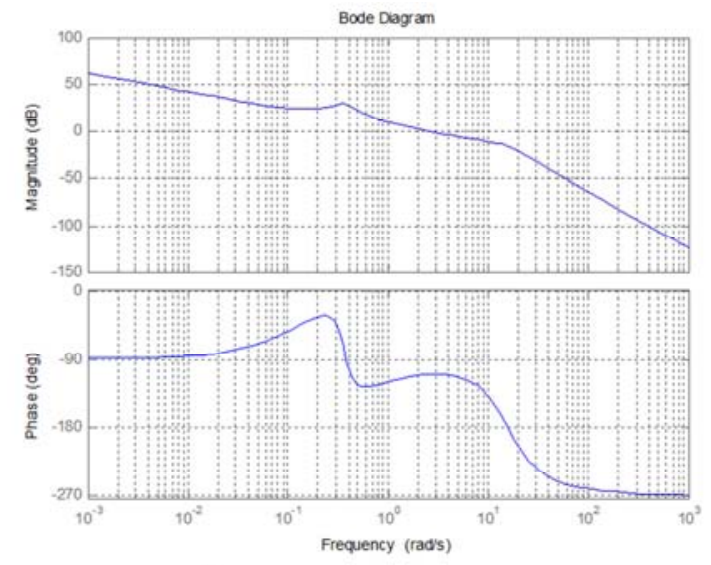

with control augment system:

$$
\begin{aligned}
\mathrm{Gm} & =16.1 \mathrm{~dB} \\
\mathrm{Pm} & =70.2^{\circ}
\end{aligned}
$$

Figure 2. Frequency-domain stability comparison.

From the comparison above, the UAV with the control augment system gets a better the time-domain and frequency-domain performance. So the flying performance of the UAV with the control augment system is much better.

\section{Longitudinal Flying Quality Evaluation}

We choose the design point $(\mathrm{h}=1000 \mathrm{~m}, \mathrm{Ma}=0.05$ ) to evaluate the flying quality of the example UAV. We evaluate the applicability of human flying quality criterion to the UAV through analyzing the longitudinal flying quality of the example UAV with and without control augment system by using human flying quality criterion. From the analysis and conclusion of the references [3-6], we evaluate the longitudinal flying quality of the example UAV by using the two typical human flying quality criteria: CAP criterion and bandwidth criterion.

\subsection{CAP Criterion Evaluation [4] [8]}

The quality level of the CAP criterion is determined by the following three parameters: equivalent short period frequency $\left(\omega_{s p}\right)$, damping ratio $\left(\zeta_{s p}\right)$ and stable vertical overload per attack angle $(\mathrm{n} / \mathrm{a})$. The equivalent short period frequency and 
damping ratio can be calculated through equivalent system method [4] [8]. Using the CAP criterion, we can get the longitudinal flying quality results of the example UAV with and without control augment system, as "Table 1" and "Figure 3" displaying.

Table 1. Flying quality results using CAP criterion.

\begin{tabular}{lllll}
\hline & $\omega_{s p} /(\mathbf{r a d} / \mathbf{s})$ & $\boldsymbol{\zeta}_{s p}$ & n/a & level \\
\hline unaugment & 7.7 & 0.74 & 8.9 & 2 \\
augment & 10.9 & 0.95 & 8.9 & 3 \\
\hline
\end{tabular}

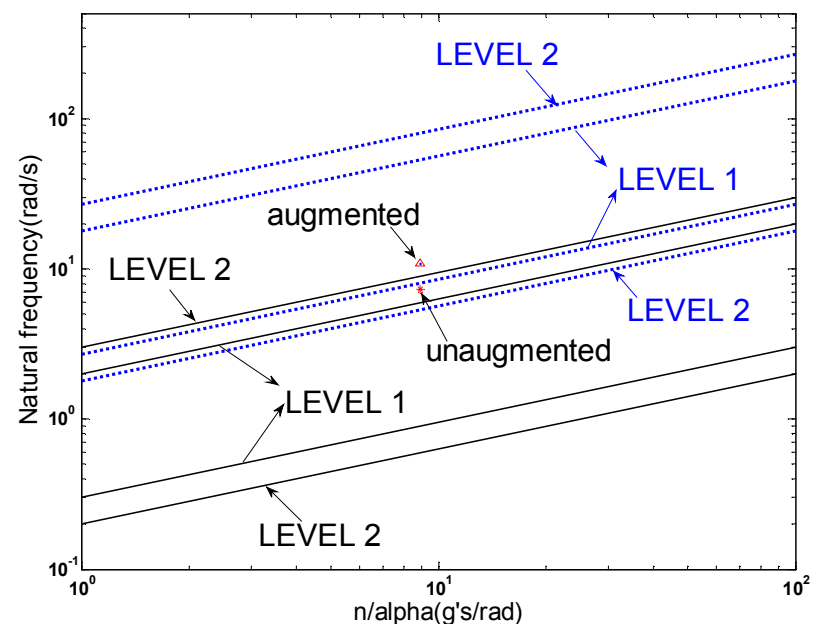

Note:Black line is CAP criterion, and blue line is the revised CAP criterion.

Figure 3. Human flying quality criterion (Phase B).

As we can see from the "Table 1" and "Figure 3", according to the human flying quality CAP criterion, the unaugmented example UAV is in level 2, but the augmented example UAV is in level 3. With the control augment system, the longitudinal flying quality of the example UAV becomes worse. But according to the performance comparison of time and frequency domains above, the flying performance of the augmented example UAV is much better than the unaugmented. So, the flying quality evaluation result based on the human CAP criterion is not consistent with the actual fact.

\subsection{Bandwidth Criterion Evaluation}

The quality level of the bandwidth criterion is determined by the following three parameters: open loop system bandwidth $\left(\omega_{\mathrm{Bw}}\right)$ and time delay $\left(\tau_{\mathrm{p}}\right)$. Firstly, bandwidth criterion usually applies to high-order system and does not require the minimum closed-loop pitch attitude bandwidth, so this criterion is applicable to different size aircrafts; Secondly, there are not any restrictions about system response properties, so this criterion can be applicable to the UAV because their responses are always changeful. It is recommended to use bandwidth criterion to evaluate the flying quality of UAV according to references of [3-5] [9]. Through comprehensive analysis, the bandwidth criterion of human flying quality is more applicable to UAV flying quality evaluation. So this paper uses bandwidth to evaluate flying quality of the example UAV.
With reference to the bandwidth criterion, we evaluate longitudinal flying quality of the example UAV at the selected point $(\mathrm{h}=2000 \mathrm{~m}, \mathrm{Ma}=0.05)$. The evaluation result is in "Table 2 ".

Table 2. Flying quality results using bandwidth criterion.

\begin{tabular}{|c|c|c|c|}
\hline & Bandwidth $\omega_{B w} /(\mathrm{rad} / \mathrm{s})$ & Time delay $\tau_{p} / \mathbf{s}$ & level \\
\hline unaugment & 5.08 & 0.0372 & 2 \\
\hline augment & 9.08 & 0.0306 & 1 \\
\hline
\end{tabular}

From "Table 2", the example UAV with control augment system gets improved flying quality, and this is consistent with the above time and frequency-domain analysis. So using bandwidth criterion to evaluate the UAV flying quality is reasonable and feasible.

\section{Flying Quality Results Analysis}

From the analysis above, the longitudinal flying quality evaluation results based on CAP criterion and bandwidth criterions are different. Combining the contrastive analysis of the time and frequency-domain, we can conclude that human CAP criterion is not applicable to the small example UAV. The small UAVs always have following features: lightweight, small size, small moment of inertial, and low speed. Refer to the following formula:

$$
\begin{aligned}
& \omega_{s p} \approx \sqrt{\frac{Z_{a} M_{\mathrm{q}}}{U_{1}}-M_{a}} \\
& M_{a}=\frac{Q S_{W} \mathrm{c}_{\mathrm{A}} C_{m a}}{I_{\mathrm{yy}}}
\end{aligned}
$$

We can see that short period frequency $\left(\omega_{s p}\right)$ is related to $M_{a}$ and velocity $U_{1}$, and $M_{a}$ is inversely proportional to moment of inertial. The short period frequency of small UAVs is always high because of their small moment of inertial and low speed. CAP criterion is not applicable to small UAVs because of their high short period frequency. Given this reason, we propose an improved CAP criterion to apply to small UAVs. The improved CAP criterion adjusts the quality levels of CAP criterion using proportional coefficient $\mathrm{N}$, and the scale relation is as following:

$$
\omega_{\text {small-scale }}=\omega_{\text {large-scale }} \sqrt{N}
$$

The coefficient $\mathrm{N}$ is the ratio of selected standard big plane wingspan and small UAV wingspan, $\mathrm{N}=80$. Based on the selected coefficient $\mathrm{N}$, we get the improved CAP criterion. According to the improved CAP criterion, the flying quality result of the example UAV is as following "Table 3" and "Figure 3":

Table 3. Flying quality results using improved CAP criterion.

\begin{tabular}{lllll}
\hline & $\omega_{s p} /(\mathbf{r a d} / \mathbf{s})$ & $\zeta_{s p}$ & n/a & level \\
\hline unaugment & 7.7 & 0.74 & 8.9 & 2 \\
augment & 10.9 & 0.95 & 8.9 & 1 \\
\hline
\end{tabular}


From "Table 3" and "Figure 3", according to the improved CAP criterion, the flying quality of example UAV without control augment system is in level 2, and in level 1 after being augmented. The example UAV with control augment system gets an improved flying quality, and this is consistent with the above time and frequency-domain analysis. So the improved CAP criterion can be applicable to the small UAV in evaluating longitudinal flying quality.

Based on all the analysis above, we can use bandwidth criterion and improved CAP criterion to evaluate the longitudinal flying quality of small UAVs.

\section{Conclusion}

This paper validates the applicability of some human flying quality criterions to small UAVs through comparing the flying performance of the example UAV with and without control augment system, and then proposes two longitudinal flying quality criterions of small UAVs: bandwidth criterion and improved CAP criterion. These two criterions present a newest idea and a useful trial to the research of UAV flying quality. And the research of this paper is useful for the design of small UAV as well as its control system design. But both these two criterions proposed by this paper have certain deficiency in analyzing flying quality of small UAVs, such as the definition of criterion boundary, so we still need a large number of fly tests to validate the applicability of these two proposed criterions to UAV flying quality evaluation.

\section{References}

[1] Ning, Dai, Hui, Yang. Flying quality specification research of UAV, Flight Dynamics journal, 2005, 23(4): 13-15.
[2] Yu jin, Tao, Jian pei, Wang. Flying quality standard research of UAV, Flight Dynamics journal, 2010, 28(1): 13-15.

[3] Feng, Wang, Sheng jun, Qi. Applicability research of Some typical flying quality to UAV, Flight Dynamics journal, 2013, 31(5): 389-3935.

[4] Cheng tao, Huang, Li xin, Wang. Longitudinal flying quality evaluation of UAV under remote mode, Flight Dynamics journal, 2013, 39(4): 427-431.

[5] M. Christopher Cotting. Applicability of Human Flying Qualities Requirements for UAVs, Finding A Way Forward [C] 27th AIAA Atmospheric Flight Mechanics Conference and Exhibit, 2009: 1-18.

[6] Holmberg, J., and King, D. J., Flying Qualities Specifications and Design Standards for Unmanned Aerial Vehicles, AIAA-2008-6555.

[7] Jin yuan, Gao, Lu yu, Li. Aircraft flying quality, 2003.

[8] Foster, T., M. and Bowman, J. W. Dynamic Stability and Handling Qualities of Small Unmanned Aerial Vehicles, AIAA-2005-1023.

[9] Giorgio Guglieri, Barbara Pralio, Fulvia Quagliotti. Flight control system design for a micro aerial vehicle [J]. Engineering \& Aerospace Technology, 2006,78(2): 87-97.

[10] Zi quan, Zhou. Flying test engineering, 2010.

[11] Orkun Simsek, Ozan Tekinalp. System Identification and Handling Quality Analysis of a UAV from Flight Test Data, AIAA-2015-1480.

[12] Elisa Capello, Giorgio Guglieri. Preliminary assessment of flying and handling qualities for mini-UAVs, Journal of Intelligent \& Robotic Systems, 2012. 